\title{
Lagrangian H-Umbilical Surfaces in Complex Lorentzian Plane
}

\author{
Shangrong Deng
}

(Communicated by Young-Ho Kim)

\begin{abstract}
We completely classify Lagrangian H-umbilical Surfaces with $\lambda=2 \mu$ in Complex Lorentzian Plane $\mathrm{C}_{1}^{2}$.

Keywords: Lagrangian submanifold, H-umbilical submanifold, Complex Lorentzian plane

AMS Subject Classification (2010): Primary 53C40, 53C15, 53C25.

\section{Introduction}

Let $L: M \rightarrow \mathbf{C}^{\mathbf{n}}$ be a Lagrangian isometric immersion. For $n \geq 3$, $\mathrm{L}$ is a Lagrangian H-umbilical immersion with $\lambda=2 \mu \neq 0$ if and only if L is locally a Lagrangian pseudo-sphere [Theorem 3.1 in [2]] .

The situation in $n=2$ is very different. Lagrangian H-umbilical Surfaces with $\lambda=2 \mu$ in complex Euclidean plane consist of a much bigger famliy of surfaces including the Lagrangian pseudo-sphere ( see [3] ).

In [4] B.-Y. Chen proved that for $n \geq 3$, $\mathrm{L}$ is a Lagrangian $\mathrm{H}$-umbilical submanifold with $\lambda=2 \mu \neq 0$ in the indefinite complex Euclidean space $\mathbf{C}_{\mathbf{k}}^{\mathbf{n}}$ if and only if $\mathbf{L}$ is locally either a Lagrangian pseudo-Riemannian sphere or a Lagrangian pseudo-hyperbolic space [Theorem 4.1 in [4]] .

In this article, we completely classify Lagrangian H-umbilical surfaces with $\lambda=2 \mu$ in complex Lorentzian plane $\mathbf{C}_{1}^{2}$. Similar to Riemanian case, Lagrangian $\mathrm{H}$-umbilical surfaces with $\lambda=2 \mu \neq 0$ in complex Lorentzian plane come from two large families of surfaces containing Lagrangian pseudo-Riemannian 2 sphere and Lagrangian pseudo-hyperbolic 2 space. Our results complete the classification of Lagrangian H-umbilical submanifolds with $\lambda=2 \mu$ in indefinite complex Euclidean spaces.
\end{abstract}

\section{Preliminaries}

Let $L: M \rightarrow \mathbf{C}_{1}^{2}$ be an isometric immersion of a 2-dimensional pseudo-Riemannian manifold $M$ into the complex Lorentzian plane $\mathbf{C}_{1}^{2}$. Then $M$ is called a Lagrangian (or totally real) submanifold if the almost complex structure $J$ of $\mathbf{C}_{1}^{2}$ carries each tangent space of $M$ into its corresponding normal space. The formulas of Gauss and Weingarten are given respectively by

$$
\begin{aligned}
& \tilde{\nabla}_{X} Y=\nabla_{X} Y+h(X, Y), \\
& \tilde{\nabla}_{X} \xi=-A_{\xi} X+D_{X} \xi,
\end{aligned}
$$

for tangent vector fields $X$ and $Y$ and normal vector fields $\xi$, where $D$ is the normal connection. The second fundamental form $h$ is related to $A_{\xi}$ by

$$
\langle h(X, Y), \xi\rangle=\left\langle A_{\xi} X, Y\right\rangle .
$$

The mean curvature vector of $M$ in $\mathbf{C}_{1}^{2}$ is defined by

$$
H=\frac{1}{2} \text { trace } h
$$


The Gauss and Codazzi equations are given by

$$
\begin{aligned}
& \langle R(X, Y) Z, W\rangle=\langle h(X, W), h(Y, Z)\rangle-\langle h(X, Z), h(Y, W)\rangle, \\
& (\nabla h)(X, Y, Z)=(\nabla h)(Y, X, Z),
\end{aligned}
$$

where $(\nabla h)$ is defined by

$$
(\nabla h)(X, Y, Z)=D_{X} h(Y, Z)-h\left(\nabla_{X} Y, Z\right)-h\left(Y, \nabla_{X} Z\right) .
$$

When $M$ is a Lagrangian surface in $\mathbf{C}_{1}^{2}$, we have

$$
\begin{aligned}
& D_{X} J Y=J \nabla_{X} Y, \\
& \langle h(X, Y), J Z\rangle=\langle h(Y, Z), J X\rangle=\langle h(Z, X), J Y\rangle .
\end{aligned}
$$

It is well known that there exist no totally umbilical Lagrangian submanifolds in a complex or psuedo complex space-form with $n \geq 2$ except the totally geodesic ones (see [7]). To investigate the "simplest" Lagrangian submanifolds next to the totally geodesic ones in complex or psuedo complex space-forms, B.-Y. Chen introduced the concept of Lagrangian H-umbilical submanifolds (cf. [2, 4]).

If $L: M \rightarrow \mathbf{C}_{1}^{2}$ is a Lagrangian $\mathrm{H}$-umbilical surface, the second fundamental form takes the following form:

$$
h\left(e_{1}, e_{1}\right)=\lambda J e_{1}, \quad h\left(e_{1}, e_{2}\right)=\mu J e_{2}, \quad h\left(e_{2}, e_{2}\right)=-\mu J e_{1}
$$

for some suitable functions $\lambda$ and $\mu$ with respect to some suitable orthonormal local frame field.

We also need the following lemma (Lemma 2.3 in [6]) in section 3.

Lemma 2.1. Let $u, v$ be any two vectors in $\mathbf{C}_{1}^{2}$ and let $a, b$ be any two complex numbers. Then we have

$$
\begin{aligned}
& \langle a u, b v\rangle=\langle a, b\rangle\langle u, v\rangle+\langle i a, b\rangle\langle u, i v\rangle, \\
& \langle a u, i b v\rangle=\langle a, b\rangle\langle u, i v\rangle+\langle a, i b\rangle\langle u, v\rangle,
\end{aligned}
$$

where $\langle a, b\rangle$ and $\langle u, v\rangle$ are cononical product for complex numbers and cononical inner product for vectors in $\mathbf{C}_{1}^{2}$.

\section{Lagrangian $\mathbf{H}$-umbilical Surfaces with $\lambda=2 \mu$ in $\mathbf{C}_{1}^{2}$}

Let $L: M \rightarrow \mathbf{C}_{1}^{2}$ be a Lagrangian H-umbilical surface with $\lambda=2 \mu$. Since the complex structure J interchanges the tangent and normal spaces of $M$ in $\mathbf{C}_{1}^{2}, M$ has real index 1 (i.e. $M$ is Lorentzian [1] or [6] ).

Theorem 3.1. Let $L: M \rightarrow \mathbf{C}_{1}^{2}$ be a Lagrangian H-umbilical surface with $\lambda=2 \mu$. If $\lambda=2 \mu=0, L$ is an open portion of a totally geodesic Lagrangian plane in $\mathbf{C}_{1}^{2}$.

Proof. If $\lambda=2 \mu=0$, the second fundamental form vanishes identically, then $M$ is totally geodesic and flat. Therefore $\mathrm{L}$ must be an open portion of a totally geodesic Lagrangian plane in $\mathbf{C}_{1}^{2}$.

From now on we assume that $\lambda=2 \mu \neq 0$.

Theorem 3.2. The following two statements hold:

(i) Let $\mu=b$ be a nonzero function, $\theta(t)$ a function on $(\alpha, \beta)$ containg 0 , and $z(t) a \mathbf{C}_{1}^{2}$ valued solution to the ordinary differential equation:

$$
z^{\prime \prime}(t)-i \theta^{\prime}(t) z^{\prime}(t)-b^{2} z(t)=0
$$

(i-a) If $z(t)$ satisfies the two conditions: $|z(t)|^{2}=-1 /\left(4 b^{2}\right)$ and $\left|z^{\prime}(t)\right|^{2}=1 / 4$, the map

$$
L(s, t)=e^{2 i b s} z(t)+\int_{0}^{t} z^{\prime}(t) e^{-2 i \theta(t)} d t
$$

defines a Lagrangian H-umbilical surface in $\mathbf{C}_{1}^{2}$ whose induced metric is

$$
g=-d s^{2}+\cos ^{2}(b s+\theta(t)) d t^{2}
$$


(i-b) If $z(t)$ satisfies the two conditions: $|z(t)|^{2}=1 /\left(4 b^{2}\right)$ and $\left|z^{\prime}(t)\right|^{2}=-1 / 4$, the map

$$
W(s, t)=e^{2 i b s} z(t)+\int_{0}^{t} z^{\prime}(t) e^{-2 i \theta(t)} d t
$$

defines a Lagrangian $H$-umbilical surface in $\mathbf{C}_{\mathbf{1}}^{\mathbf{2}}$ whose induced metric is

$(3.3-b)$

$$
g=d s^{2}-\cos ^{2}(b s+\theta(t)) d t^{2}
$$

and in either case the second fundamental form is

$$
h\left(e_{1}, e_{1}\right)=2 b J e_{1}, \quad h\left(e_{1}, e_{2}\right)=b J e_{2}, \quad h\left(e_{2}, e_{2}\right)=-b J e_{1}
$$

with respect to $e_{1}=\partial / \partial s$ and $e_{2}=\sec (b s+\theta(t)) \partial / \partial t$.

(ii) Conversely, if $\tilde{L}: M \rightarrow \mathbf{C}_{1}^{2}$ is a Lagrangian H-umbilical surface whose second fundamental form satisfies (3.4) for some function $b \neq 0$ with respect to some orthonormal local frame field $\left\{e_{1}, e_{2}\right\}$, then we have:

(ii-1) $b$ is a constant

(ii-2) there exist a function $\theta(t)$ and a local coordinate system $\{s, t\}$ on $M$ with $e_{1}=\partial / \partial s$ such that the metric tensor is given by $(3.3-a)$ (or $(3.3-b))$, and

(ii-3) the immersion $\tilde{L}$ is congruent to the $L$ ( or $W$ respectively ) given in statement $(i)$.

Proof. $(i)$ :

Case $(i-a)$ : Consider the map defined by $(3.2-a)$ satisfying $(3.1)$ and $|z(t)|^{2}=-1 /\left(4 b^{2}\right)$ and $\left|z^{\prime}(t)\right|^{2}=1 / 4$. Then we have

$$
\begin{aligned}
& L_{s}=2 i b e^{2 i b s} z(t), \quad L_{t}=\left(e^{2 i b s}+e^{-2 i \theta}\right) z^{\prime}(t), \\
& L_{s s}=-4 b^{2} e^{2 i b s} z(t), \quad L_{s t}=2 i b e^{2 i b s} z^{\prime}(t), \\
& L_{t t}=\left(e^{2 i b s}+e^{-2 i \theta}\right) z^{\prime \prime}(t)-2 i e^{-2 i \theta} \theta^{\prime} z^{\prime}(t) .
\end{aligned}
$$

By direct computation and (3.1) we also have

$$
\begin{aligned}
& L_{s s}=2 i b L_{s}, \\
& L_{s t}=i b \sec (b s+\theta) e^{i(b s+\theta)} L_{t} \\
& L_{t t}=-i b \cos (b s+\theta) e^{-i(b s+\theta)} L_{s}-\theta^{\prime} \tan (b s+\theta) L_{t} .
\end{aligned}
$$

Solution of $(3.1) z(t)$ satisfies $\left\langle z^{\prime}, i z\right\rangle=0$. Using Lemma 2.1 we have

$$
\begin{aligned}
& \left\langle L_{s}, L_{s}\right\rangle=-1, \quad\left\langle L_{s}, L_{t}\right\rangle=0 . \\
& \left\langle L_{t}, L_{t}\right\rangle=\cos ^{2}(b s+\theta), \\
& \left\langle i L_{s}, L_{s}\right\rangle=0, \quad\left\langle i L_{s}, L_{t}\right\rangle=0 \\
& \left\langle i L_{t}, L_{s}\right\rangle=0, \quad\left\langle i L_{t}, L_{t}\right\rangle=0
\end{aligned}
$$

Therefore $L$ is Lagrangian and the induced metric is given by $(3.3-a)$, from which we have

$$
\begin{aligned}
& \nabla_{\partial / \partial s \frac{\partial}{\partial s}}=0, \quad \nabla_{\partial / \partial s} \frac{\partial}{\partial t}=-b \tan (b s+\theta) \frac{\partial}{\partial t} \\
& \nabla_{\partial / \partial s \frac{\partial}{\partial s}}=-\frac{b}{2} \sin (2 b s+2 \theta) \frac{\partial}{\partial s}-\theta^{\prime} \tan (b s+\theta) \frac{\partial}{\partial t} .
\end{aligned}
$$

Setting $e_{1}=\frac{\partial}{\partial s}$ and $e_{2}=\sec (b s+\theta) \frac{\partial}{\partial t}$, from Gauss formula we have

$$
h\left(\frac{\partial}{\partial s}, \frac{\partial}{\partial s}\right)=L_{s s}-\nabla_{\partial / \partial s} \frac{\partial}{\partial s}=2 b i L_{s}=2 b J \frac{\partial}{\partial s}
$$

which is

$$
h\left(e_{1}, e_{1}\right)=2 b J e_{1} .
$$

Similarly from (3.6), (3.7), and Gauss formula

$$
h\left(e_{1}, e_{2}\right)=b J e_{2}, \quad h\left(e_{2}, e_{2}\right)=-b J e_{1} .
$$


Therefore $L$ is a Lagrangian $\mathrm{H}$-umbilical surface with $\lambda=2 \mu=2 b$ in complex Lorentzian plane $\mathbf{C}_{\mathbf{1}}^{2}$

Case $(i-b)$ : it can be proved using the same method as in Case $(i-a)$ with minor modification.

(ii):

Conversely, assume that $\tilde{L}: M \rightarrow \mathbf{C}_{1}^{2}$ is a Lagrangian H-umbilical surface whose second fundamental form satisfies (3.4) for some function $b \neq 0$ with respect to some orthonormal local frame field $\left\{e_{1}, e_{2}\right\}$. Since $M$ has real index 1 , we divide the proof into two cases: $e_{1}$ is time-like or $e_{1}$ is space-like:

Case $(i i-a): e_{1}$ is time-like. We may assume

$$
\left\langle e_{1}, e_{1}\right\rangle=-1, \quad\left\langle e_{1}, e_{2}\right\rangle=0, \quad\left\langle e_{2}, e_{2}\right\rangle=1
$$

Since $M$ is H-umbilical, we have the following Codazzi and Gauss equations (see p.166 in [4] ):

$$
\begin{aligned}
& e_{1} \mu=(\lambda-2 \mu) \omega_{1}^{2}\left(e_{2}\right), \\
& e_{2} \lambda=(2 \mu-\lambda) \omega_{1}^{2}\left(e_{1}\right), \\
& e_{2} \mu=-3 \mu \omega_{1}^{2}\left(e_{1}\right), \\
& K=\mu(\mu-\lambda) .
\end{aligned}
$$

Since $\lambda=2 \mu=2 b \neq 0$, from (3.8) we have that $\mathrm{b}$ is constant, $K=-b^{2}$ and $\omega_{1}^{2}\left(e_{1}\right)=0$. Therefore $\nabla_{e_{1}} e_{1}=0$ and the integral curves of $e_{1}$ are geodesic in $M$. Thus, there exists a local cordinate system $\{s, u\}$ such that $e_{1}=\partial / \partial s$ and the metric tensor of $M$ is $g=-d s^{2}+f^{2}(s, u) d u^{2}$. The Gauss curvature $\mathrm{K}$ of $M$ is given by ( $\mathrm{p} .81$ in [8] ):

$$
K=f_{s s} / f
$$

Therefore we have $f_{s s}+b^{2} f=0$. Solving this equation yields

$$
f=A(u) \cos (b s)+B(u) \sin (b s)
$$

for some functions $A(u)$ and $B(u)$. Thus, we have

$$
g=-d s^{2}+r^{2}(u) \cos ^{2}(b s+\phi(u)) d u^{2}
$$

where $A(u)=r(u) \cos \phi(u), B(u)=r(u) \sin \phi(u)$ and $r(u)=\sqrt{A^{2}+B^{2}}$. If we set $t=t(u)$ an antiderivative of $r(u),(3.9)$ becomes

$$
g=-d s^{2}+\cos ^{2}(b s+\theta(t)) d t^{2}
$$

for some function $\theta(t)$. From (3.10) we have

$$
\begin{aligned}
& \nabla_{\partial / \partial s} \frac{\partial}{\partial s}=0, \quad \nabla_{\partial / \partial s} \frac{\partial}{\partial t}=-b \tan (b s+\theta) \frac{\partial}{\partial t}, \\
& \nabla_{\partial / \partial s \frac{\partial}{\partial s}}=-\frac{b}{2} \sin (2 b s+2 \theta) \frac{\partial}{\partial s}-\theta^{\prime} \tan (b s+\theta) \frac{\partial}{\partial t} .
\end{aligned}
$$

From (3.4), (3.10), (3.11) and Gauss formula we see that the immersion satisfies the following system of PDEs:

$$
\begin{aligned}
& \tilde{L}_{s s}=2 i b \tilde{L}_{s}, \\
& \tilde{L}_{s t}=i b \sec (b s+\theta) e^{i(b s+\theta)} \tilde{L}_{t} \\
& \tilde{L}_{t t}=-i b \cos (b s+\theta) e^{-i(b s+\theta)} \tilde{L}_{s}-\theta^{\prime} \tan (b s+\theta) \tilde{L}_{t} .
\end{aligned}
$$

Solving the first two equations in (3.12) we get

$$
\tilde{L}=A(t) e^{2 i b s}+B(t)
$$

for some $\mathbf{C}_{\mathbf{1}}^{2}$ valued functions $A(t), B(t)$. Differentiating (3.13) yields

$$
\tilde{L}_{t}=A^{\prime}(t) e^{2 i b s}+B^{\prime}(t) \text {. }
$$


Substituting (3.14) into the second equation of (3.12), we have

$$
B^{\prime}(t)=e^{-2 i \theta} A^{\prime}(t)
$$

Combining (3.14) and (3.15) gives

$$
\tilde{L}_{t}=A^{\prime}(t)\left(e^{2 i b s}+e^{-2 i \theta}\right)
$$

After a suitable translation, we find that

$$
\tilde{L}=e^{2 i b s}(A(t)+C)+\int_{0}^{t} A^{\prime}(t) e^{-2 i \theta(t)} d t
$$

for some constant vector $C$. Therefore, if we put $z(t)=(A(t)+C)$, we obtain $(3.2-a)$.

$(3.18$ or $3.2-a)$

$$
\tilde{L}=e^{2 i b s} z(t)+\int_{0}^{t} z^{\prime}(t) e^{-2 i \theta(t)} d t
$$

Substituting (3.18) into the last equation in (3.12) we find

$$
z^{\prime \prime}(t)-i \theta^{\prime}(t) z^{\prime}(t)-b^{2} z(t)=0
$$

Therefore $z(t)$ is a solution to (3.1). From (3.18) we have

$$
\tilde{L}_{s}=2 i b e^{2 i b s} z(t) \quad \tilde{L}_{t}=\left(e^{2 i b s}+e^{-2 i \theta}\right) z^{\prime}(t)
$$

which implies that $\left|\tilde{L}_{s}\right|^{2}=4 b^{2}|z(t)|^{2}$ and $\left|\tilde{L}_{t}\right|^{2}=4 \cos ^{2}(b s+\theta)\left|z^{\prime}(t)\right|^{2}$. Comparing these with (3.10) gives $|z(t)|^{2}=-1 /\left(4 b^{2}\right)$ and $\left|z^{\prime}(t)\right|^{2}=1 / 4$. By the uniqueness theorem in [4], the immersion $\tilde{L}$ is congruent to the immersion $L$ defined in statement $(i)$ if $e_{1}$ is time-like.

Case $(i i-b): e_{1}$ is space-like. We may assume

$$
\left\langle e_{1}, e_{1}\right\rangle=1, \quad\left\langle e_{1}, e_{2}\right\rangle=0, \quad\left\langle e_{2}, e_{2}\right\rangle=-1
$$

Since $M$ is H-umbilical, we have the following Codazzi and Gauss equations (see p.174 in [4] ):

$$
\begin{aligned}
& e_{1} \mu=-(\lambda-2 \mu) \omega_{1}^{2}\left(e_{2}\right), \\
& e_{2} \lambda=-(2 \mu-\lambda) \omega_{1}^{2}\left(e_{1}\right), \\
& e_{2} \mu=3 \mu \omega_{1}^{2}\left(e_{1}\right), \\
& K=\mu(\lambda-\mu) .
\end{aligned}
$$

Since $\lambda=2 \mu=2 b \neq 0$, from (3.21) we have that $\mathrm{b}$ is constant, $K=b^{2}$ and $\omega_{1}^{2}\left(e_{1}\right)=0$. Therefore $\nabla_{e_{1}} e_{1}=0$ and the integral curves of $e_{1}$ are geodesic in $M$. Thus, there exists a local cordinate system $\{s, u\}$ such that $e_{1}=\partial / \partial s$ and the metric tensor of $M$ is $g=d s^{2}-f^{2}(s, u) d u^{2}$. But the Gauss curvature K of $M$ is given by ( p.81 in [8] ):

$$
K=-f_{s s} / f
$$

Therefore we have $f_{s s}+b^{2} f=0$. The rest of the proof is almost the same as in Case $(i i-a)$. Finally we see that the immersion $\tilde{L}$ is congruent to the immersion $W$ defined in statement $(i)$ if $e_{1}$ is space-like.

For the existence of solution to (3.1) subject to the two conditions in $(i-a)$ in Theorem 3.2 , we have the following

Theorem 3.3. For any nonzero number $b$ and any differentiable function $\theta$ of one variable defined on interval $I$, there exists a $\mathbf{C}_{1}^{2}$ valued solution to the differential equation:

$$
z^{\prime \prime}(t)-i \theta^{\prime}(t) z^{\prime}(t)-b^{2} z(t)=0
$$

that also satisfies the two conditions: $|z(t)|^{2}=-1 /\left(4 b^{2}\right)$ and $\left|z^{\prime}(t)\right|^{2}=1 / 4$. 
Proof. Let $b$ be a nonzero number $b$ and any differentiable function $\theta$ of one variable defined on interval $I$. Let $D$ be a simply connected open domain in $R^{2}=\{(s, t) \mid s, t \in R\}$ on which the function $\cos (b s+\theta(t))$ is nowhere zero.

Let $M=(D, g)$ be the Semi -Riemannian 2-manifold equipped with metric tensor $g=-d s^{2}+\cos ^{2}(b s+$ $\theta(t)) d t^{2}$. Then its Levi-Civita connection satisfies (3.11).

Now we form a $T M$-valued symmetric bilinear form such that

$$
\begin{aligned}
& \sigma\left(\frac{\partial}{\partial s}, \frac{\partial}{\partial s}\right)=2 b \frac{\partial}{\partial s}, \quad \sigma\left(\frac{\partial}{\partial s}, \frac{\partial}{\partial t}\right)=b \frac{\partial}{\partial t}, \\
& \sigma\left(\frac{\partial}{\partial t}, \frac{\partial}{\partial t}\right)=-b \cos ^{2}(b s+\theta(t)) \frac{\partial}{\partial s}
\end{aligned}
$$

Then $\langle\sigma(X, Y), Z\rangle$ and $(\nabla \sigma)(X, Y, Z)=\nabla_{X} \sigma(Y, Z)-\sigma\left(\nabla_{X} Y, Z\right)-\sigma\left(Y, \nabla_{X} Z\right)$ are totally symmetric. A direct computation shows that the curvature tensor of $M$ satisfies $R(X, Y) Z=\sigma(\sigma(Y, Z), X)-\sigma(\sigma(X, Z), Y)$. By the existence theorem (p.155 in [4]), there exists a Lagrangian immersion $\tilde{L}: M \rightarrow \mathbf{C}_{1}^{2}$ whose second fundamental form $h=J \sigma$ satisfies (3.4). As in the proof of Theorem 3.2, we see that the immersion also satisfies (3.12). After solving the first two equations of (3.12) in the same way as in Theorem 3.2, we have

$$
\tilde{L}=e^{2 i b s} z(t)+\int_{0}^{t} z^{\prime}(t) e^{-2 i \theta(t)} d t+C
$$

for some $\mathbf{C}_{\mathbf{1}}^{2}$ valued function $z(t)$ and constant vector $C$. Substituting (3.24) into the last equation of (3.12), we find that

$$
z^{\prime \prime}(t)-i \theta^{\prime}(t) z^{\prime}(t)-b^{2} z(t)=0
$$

Therefore $z(t)$ is a solution to (3.22). Using (3.10) and (3.24), we see that $z(t)$ also satisfies the two conditions: $|z(t)|^{2}=-1 /\left(4 b^{2}\right)$ and $\left|z^{\prime}(t)\right|^{2}=1 / 4$.

Remark 3.1. Using the same method as in Theorem 3.3, we can also prove the existence of solution to (3.1) subject to the two conditions in $(i-b)$ in Theorem 3.2.

Remark 3.2. Theorem 3.1 and Theorem 3.2 completely classify Lagrangian $\mathrm{H}$-umbilical Surfaces with $\lambda=2 \mu$ in Complex Lorentzian Plane $\mathbf{C}_{1}^{2}$

Remark 3.3. From Theorem 3.2 and Theorem 3.3 we see that the class of Lagrangian H-umbilical Surfaces with $\lambda=2 \mu$ in Complex Lorentzian Plane $\mathbf{C}_{1}^{2}$ is very large. This is different from higher dimensional cases. If $\theta=0$, the immersion becomes a Lagrangian pseudo-Riemannian 2 sphere or a Lagrangian pseudo-hyperbolic 2 space in $\mathbf{C}_{1}^{2}$ as in [4].

\section{References}

[1] Anciaux, H., Minimal Submanifolds in Pseudo-Riemannian Geometry. World Scientific Publications, New Jersey, 2010.

[2] Chen, B.-Y., Complex extensors and Lagrangian submanifolds in complex Euclidean spaces. Tohoku Math. J. 49 (1997), $277-297$.

[3] Chen, B.-Y., Lagrangian Surfaces of Constant Curvature in Complex Euclidean Plane. Tohoku Math. J. 56 (2004), 289-298.

[4] Chen, B.-Y., Complex extensors and Lagrangian submanifolds in indefinite complex Euclidean spaces. Bulletin Math. Inst. Academia Sinica 31 (2003), 151-179.

[5] Chen, B.-Y., Pseudo-Riemannian Geometry, $\delta$-invariants and Applications. World Scientific Publications, Hackensack, New Jersey, 2011.

[6] Chen, B.-Y., A Construction Method of Lagrangian Surfaces in Complex Pseudo Euclidean Plane $\mathbf{C}_{1}^{2}$ and its Applications. Int. Electron. J. Geom. 7 (2014), 4-25.

[7] Chen, B.-Y. and Ogiue, K., Two theorems on Kaehler manifolds. Michigan Math. J. 21 (1974), 225-229.

[8] B. O'Neill, Semi-Riemannian Geometry with Applications to Relativity. Academic Press, New York, 1983.

[9] K. Yano and M. Kon, Structures on manifolds, Series in Pure Mathematics, 3. World Scientific Publishing Co., Singapore, 1984. 


\section{Affiliations}

SHANGRONG DENG

ADDRESS: Department of Mathematics, Kennesaw State University, 1100 South Marietta Parkway Marietta, GA 30060, U.S.A

E-MAIL: sdeng@kennesaw.edu 